\title{
Phenotypic Variability Associated with a Large Recurrent 1q21.1 Microduplication in a Three-Generation Family
}

\author{
Judith M.A. Verhagen ${ }^{a}$ Nicole de Leeuw ${ }^{b}$ Dimitri N.M. Papatsonis ${ }^{c}$ \\ Els W.M. Grijseels ${ }^{c}$ Ronald R. de Krijger ${ }^{d}$ Marja W. Wessels ${ }^{a}$ \\ ${ }^{a}$ Department of Clinical Genetics, Erasmus Medical Center, Rotterdam, ${ }^{b}$ Department of Human Genetics, Radboud \\ University Medical Center, Nijmegen, ' Department of Obstetrics and Gynecology, Amphia Hospital, Breda, and \\ ${ }^{\mathrm{d}}$ Department of Pathology, Reinier de Graaf Gasthuis, Delft, The Netherlands
}

\section{Key Words}

1q21.1 microduplication · Congenital heart defects · Copy number variation $\cdot$ GJA5

\begin{abstract}
Recurrent copy number variants of the q21.1 region of chromosome 1 have been associated with variable clinical features, including developmental delay, mild to moderate intellectual disability, psychiatric and behavioral problems, congenital heart malformations, and craniofacial abnormalities. A subset of individuals is clinically unaffected. We describe a unique 3-generation family with a large recurrent 1q21.1 microduplication (BP2-BP4). Our observations underline the incomplete penetrance and phenotypic variability of this rearrangement. We also confirm the association with congenital heart malformations, chronic depression, and anxiety. Furthermore, we report a broader range of dysmorphic features. The extreme phenotypic heterogeneity observed in this family suggests that additional factors modify the clinical phenotype.

(c) 2015 S. Karger AG, Basel
\end{abstract}

The proximal end of the long arm of chromosome 1 is susceptible to recurrent rearrangements with clustered breakpoints (BP), resulting from non-allelic homologous recombination mediated by low-copy repeats. The chromosome 1q21.1 region can be subdivided into 2 distinctive regions. The proximal region, extending from $\mathrm{BP} 2$ to $\mathrm{BP} 3$, spans $200 \mathrm{~kb}$ (chr1:145.4-145.6 Mb, GRCh37/hg19), and the distal region, extending from BP3 to BP4, spans 1.35 Mb (chr1:146.5-147.9 Mb, GRCh37/h19). Proximal 1q21.1 microdeletions are detected in the majority of patients with thrombocytopenia-absent radius (TAR) syndrome (OMIM: 274000), in combination with 1 of 2 lowfrequency SNPs in regulatory regions of the RBM8A gene [Klopocki et al., 2007; Albers et al., 2012]. Furthermore, recurrent proximal 1q21.1 microdeletions and microduplications have been associated with failure to thrive, feeding problems, developmental delay (DD) and/or intellectual disability (ID), behavior problems (including autism and attention deficit hyperactivity disorder), congenital heart malformations, and a variety of dysmorphic features [Rosenfeld et al., 2012]. Recurrent distal 1q21.1 microdeletions (OMIM: 612474), sometimes referred to as class I deletions, are associated with DD/ID, schizophrenia, mi-

\section{KARGER 125}

(c) 2015 S. Karger AG, Base

$1661-8769 / 15 / 0062-0071 \$ 39.50 / 0$

E-Mail karger@karger.com

www.karger.com/msy
Judith M.A. Verhagen

Department of Clinical Genetics, Erasmus Medical Center

PO Box 2040

NL-3000 CA Rotterdam (The Netherlands)

E-Mail j.m.a.verhagen@erasmusmc.nl 


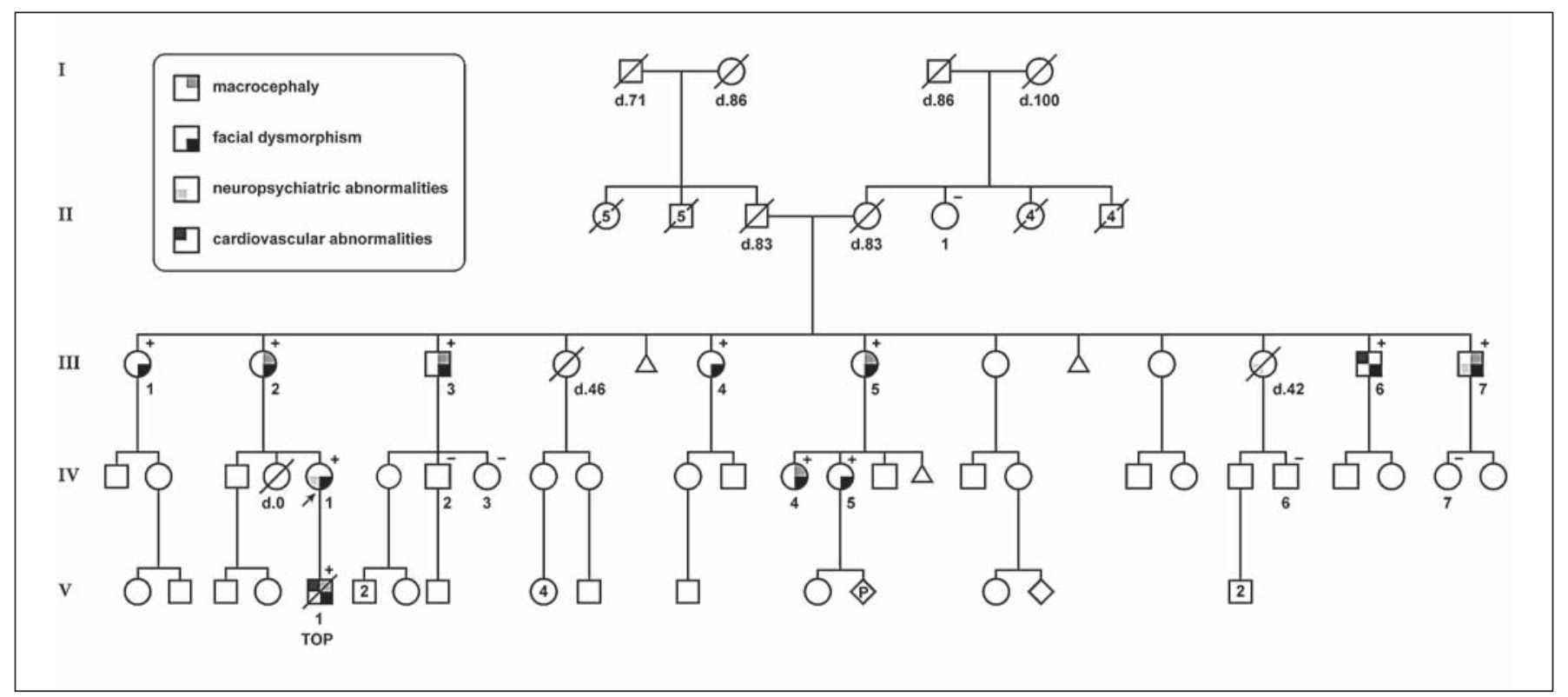

Fig. 1. Pedigree of the family with a recurrent 1q21.1 microduplication (BP2-BP4). Squares indicate males, circles indicate females. Diamonds reflect persons of unspecified gender. An arrow points to the proband. Age of death (d.) is displayed below the symbol, and TOP indicates termination of pregnancy. The presence $(+)$ or absence $(-)$ of the 1q21.1 microduplication is indicated in the upper right corner.

crocephaly, mild dysmorphic features (e.g. frontal bossing, deep-set eyes, bulbous nose), congenital heart malformations, cataracts, and joint laxity. Individuals with the reciprocal distal microduplication (OMIM: 612475) manifest DD/ID, autism, macrocephaly, and mild dysmorphic features (e.g. frontal bossing and hypertelorism) [Brunetti-Pierri et al., 2008; Mefford et al., 2008]. Finally, a third category of large (or class II) recurrent 1q21.1 microdeletions and microduplications has been identified which encompasses both the proximal and distal region. Clinical features overlap with the smaller proximal and distal rearrangements. However, as the number of individuals published to date is limited, the exact phenotypic consequences remain unclear. Here, we describe a unique 3-generation family with a large $(2.6 \mathrm{Mb})$ microduplication of chromosome 1q21.1 spanning from BP2 to BP4.

\section{Clinical Report}

The proband (IV-1, fig. 1) visited our Department of Clinical Genetics for genetic counseling. She was born at term after an uneventful pregnancy. Delivery was complicated by perinatal asphyxia, and birth weight was 4,650 g ( $>95$ th percentile). She had completed vocational education with severe learning difficulties. Since the age of 18 years she had suffered from chronic depression and anxiety. At present, she works at a sheltered workshop. Physical examination at 32 years of age revealed a tall stature $(90-95$ th percentile), broad face, full upper eyelids, narrow palpebral fissures, hypertelorism, wide mouth, and thin lips (fig. 2a). Head circumference was normal (75th percentile), and cardiologic examination revealed no abnormalities. During her first pregnancy (V-1; fig. 1), the second-trimester ultrasound examination at 20 weeks of gestation showed fetal overgrowth (parameters $>95$ th percentile) and a complex congenital heart malformation consisting of dilated ventricles with hypertrabeculated myocardium, a ventricular septal defect, and dilated main pulmonary artery and aorta. After multidisciplinary counseling, the parents decided to terminate the pregnancy. At 22 weeks and 6 days of gestation, a stillborn fetus was delivered with a birth weight of $720 \mathrm{~g}$ (>95th percentile), crown-heel length of $32.5 \mathrm{~cm}$ (75-90th percentile), and head circumference of $23 \mathrm{~cm}$ ( $>95$ th percentile). Physical examination demonstrated a male fetus with a square face, hypertelorism, short palpebral fissures, wide mouth, and thin lips (fig. 3a). Autopsy revealed a grossly enlarged heart with hypertrabeculated myocardium, absent pulmonary valve, tricuspid valve dysplasia, perimembranous ventricular septal defect, persistent left superior vena cava, and an abnormal widening and thickening of the aortic wall (fig. 3b).

\section{Materials and Methods}

Genomic DNA was extracted from peripheral blood or cultured amniocytes using the Chemagic Magnetic Separation Module I (Chemagen, BaesWeiler, Germany). Multiplex ligation-de- 

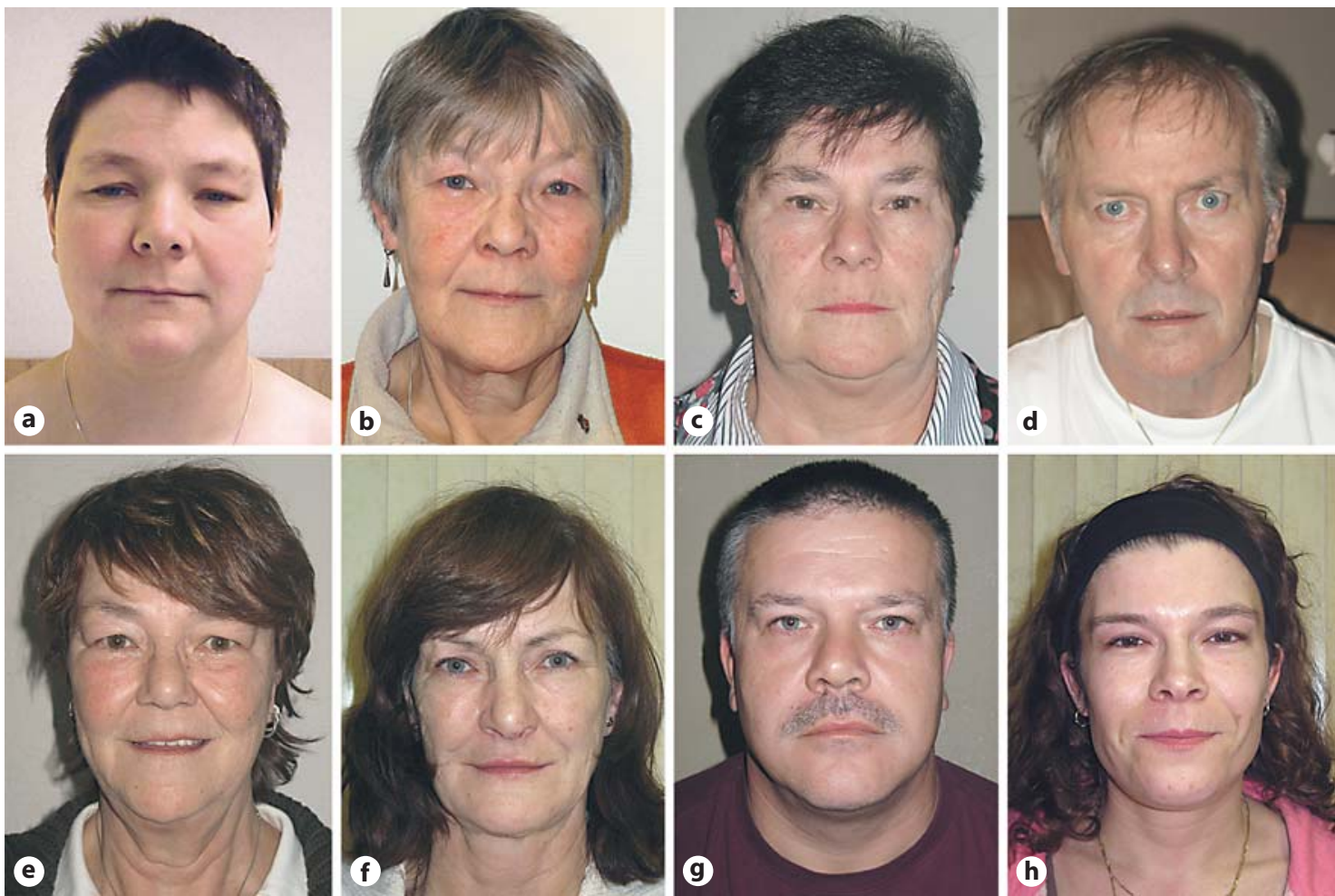

Fig. 2. Facial features of family members carrying the $1 \mathrm{q} 21.1$ microduplication. a Patient IV-1 (proband). b Patient III-2 (mother of the proband). c Patient III-1. d Patient III-3. e Patient III-4. f Patient III-5. g Patient III-7. h Patient IV-4.
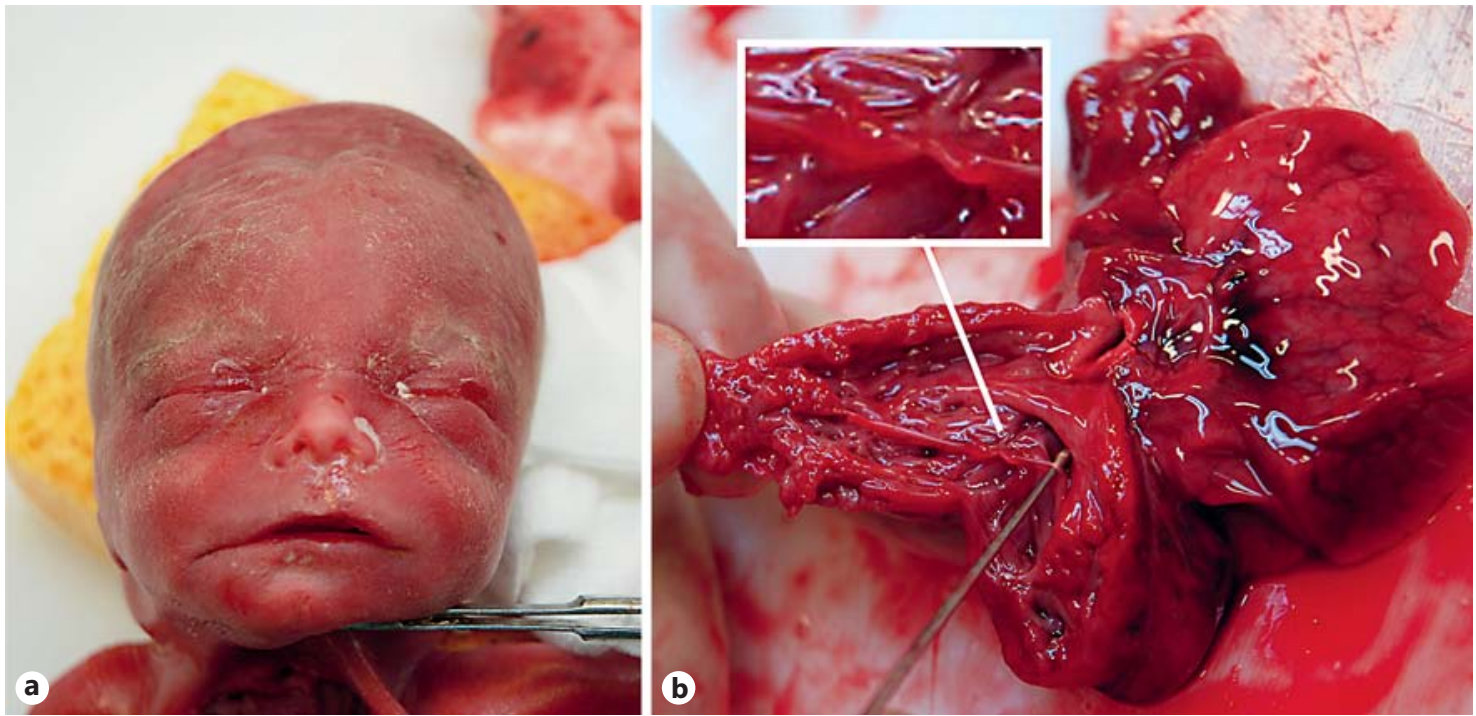

Fig. 3. a Facial features of the fetus (patient V-1). b Longitudinal section through the right ventricle showing a thickened and hypertrabeculated myocardium, the absence of a pulmonary valve, and tricuspid valve dysplasia with nodular thickening of the chordae tendineae (enlarged detail). 
Table 1. Clinical features of affected family members

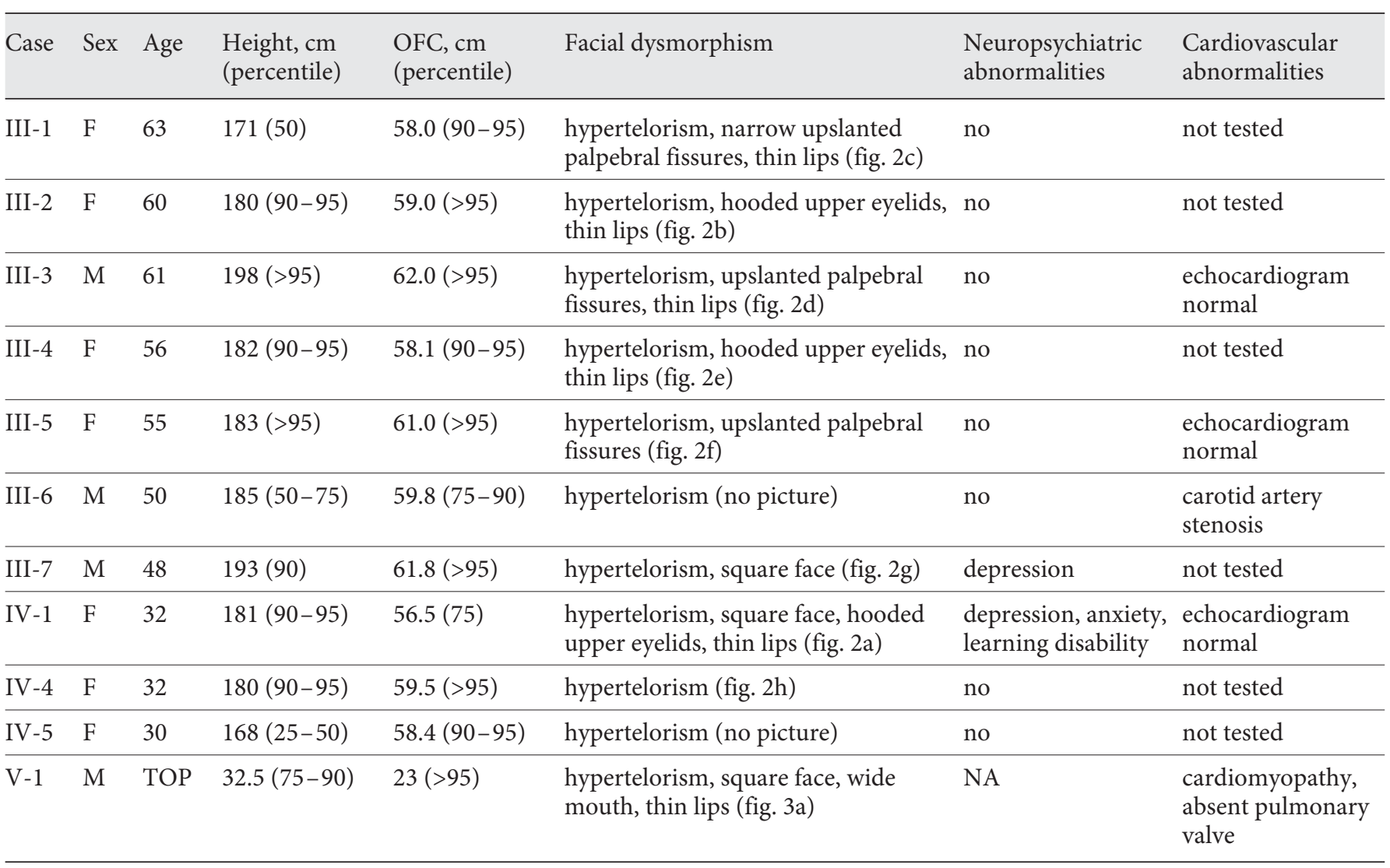

$\mathrm{NA}=$ Not applicable; $\mathrm{OFC}=$ occipitofrontal circumference; $\mathrm{TOP}=$ termination of pregnancy.

pendent probe amplification (MLPA) analysis of the 22q11 region was performed using the SALSA MLPA kit P250 DiGeorge (MRC Holland, Amsterdam, The Netherlands). Genome wide array analyses were performed on the Affymetrix GeneChip 250K NspI (Affymetrix, Santa Clara, Calif., USA) and analyzed using CNAG 2.0 software as previously described [de Leeuw et al., 2011]. Results were mapped to the human genome build CRCh37/h19. All procedures were carried out according to the manufacturer's protocols. Medical history was obtained by self-reports and/or review of medical records. Physical examination was performed by 2 clinical geneticists (J.M.A.V. and M.W.W.), and measurements were plotted on Dutch growth charts. All subjects provided written informed consent for participation in this study and (if applicable) publication of photographs.

\section{Results}

Karyotyping and MLPA analysis of the 22q11.2 region on cultured amniocytes of the fetus demonstrated no abnormalities. Genome wide array analysis revealed a 2.6
$\mathrm{Mb}$ duplication on the long arm of chromosome 1 in both the proband and her fetus: arr 1q21.1(145,243,316$147,814,694) \times 3$. This duplication encompasses $\sim 38$ protein-coding genes. The healthy mother of the proband (III-2) was also found to carry the 1q21.1 microduplication. Further studies in 13 family members identified 8 additional carriers (fig 1.). In none of the family members other (potentially) clinically relevant copy number gains or losses were identified by array analysis. In IV-2 and IV-3, who both did not carry the 1q21.1 microduplication, a $14 \mathrm{Mb}$ homozygous stretch was observed involving the pericentromeric region of chromosome 2. This is a fairly common finding. As both sibs did not display any clinical features, no additional testing was performed.

The clinical features of all carriers $(n=11)$ are summarized in table 1. All carriers displayed (mild) ocular hypertelorism. Other dysmorphic features included rectangular face, hooded upper eyelids, narrow or upslanted palpebral fissures, thin lips, and long chin (fig. 2). Macro- 
cephaly (defined as a head circumference $>95$ th percentile) was observed in 6/11 (54\%). Except for the proband, none of the other family members had DD/ID. Patient III-6 was treated for carotid artery stenosis. None had signs or symptoms of (congenital) heart disease. Of note, formal cardiologic examination including echocardiography was performed in only 3 family members (i.e. patient III-3, III-5, and IV-1). Patient III-7 also suffered from chronic depression. The non-carriers $(n=5)$ did not display any of these features. Level of functioning, estimated from the highest completed level of education and employment, fell within the range of the normal population. No marked difference was observed between carrier and non-carrier family members.

\section{Discussion}

We report on 11 family members with a large recurrent 1q21.1 microduplication (BP2-BP4). Similar duplications have been reported previously in 6 cases. Three patients presented with tetralogy of Fallot (TOF), which was accompanied by macrocephaly and neuropsychiatric features in one patient [Greenway et al., 2009; Soemedi et al., 2012; Dolcetti et al., 2013]. One patient displayed schizophrenia and learning disability [Dolcetti et al., 2013], and another patient, who also carried a $0.6-\mathrm{Mb}$ 22q11.22 duplication, had complete uterine and vaginal agenesis and fused external labia [Cheroki et al., 2008]. Clinical information was not available in one case [Brunetti-Pierri et al., 2008]. The 1q21.1 microduplication was inherited from an apparently unaffected parent in 2 patients and of unknown inheritance in the remaining 4.

A much larger number of patients with the smaller overlapping proximal (BP2-BP3) or distal (BP3-BP4) 1q21.1 microduplications have been described. Their phenotypes have been summarized by Rosenfeld et al. [2012]. The frequency of DD/ID in patients with proximal and distal 1q21.1 microduplications (75 and 78\%, respectively) was strikingly higher compared to our family (10\%). This discrepancy might reflect an overrepresentation of severely affected carriers in previous reports, as these cases are more likely to be included in genetic studies and published in the literature. Mildly affected or unaffected carriers of 1q21.1 microduplications may have remained undiagnosed. Furthermore, our findings confirm the previously reported association of 1q21.1 microduplications with congenital heart malformations, chronic depression, and anxiety [Brunetti-Pierri et al., 2008].

Familial 1q21.1 Microduplication
The dysmorphic facial features of 1q21.1 microduplications are not easily recognizable. The most consistently reported findings include (relative) macrocephaly, frontal bossing, and hypertelorism [Brunetti-Pierri et al., 2008; Mefford et al., 2008]. In our family, we observed additional features, including rectangular face, hooded upper eyelids, narrow or upslanted palpebral fissures, thin lips, and long chin.

Congenital heart malformations occur in approximately 18 and $29 \%$ of patients with proximal and distal 1q21.1 microduplications, respectively. These comprise a broad spectrum of abnormalities, including left-sided, right-sided, conotruncal, and septal defects [Digilio et al., 2013]. The prevalence of congenital heart malformations appears to be similar in the reciprocal microdeletions (17 and 26\%, respectively) [Rosenfeld et al., 2012]. These observations suggest that genes located in the distal part of the 1q21.1 region play a major role in the cardiac phenotype. The GJA5 gene, coding for the gap junction protein connexin 40 (CX40), is considered a strong candidate gene. This is based on several lines of evidence: (a) Gja5 heterozygous and homozygous knock-out mice show various cardiac malformations [Gu et al., 2003]; (b) germline and somatic mutations in GJA5 predispose to atrial fibrillation [Gollob et al., 2006; Yang et al., 2010; Wirka et al., 2011]; (c) small (100-200 kb) rare duplications encompassing only the GJA5 gene are enriched in patients with non-syndromic TOF [Soemedi et al., 2012]; (d) and lastly, a missense variant (p.Pro265Ser) at the carboxylterminus of GJA5 was detected in 2/178 patients with non-syndromic TOF. This variant was not observed in 1,568 ethnically-matched controls [Guida et al., 2012]. The hypertrabeculated myocardium observed in patient IV-1 has not been reported previously in patients with 1q21.1 microduplications. However, dilated and hypertrophic cardiomyopathy has been observed in some Gja5 homozygous mouse mutants [Kirchhoff et al., 2000; Gu et al., 2003], suggesting that GJA5 gene dosage alterations might predispose to cardiomyopathy. The PRKAB2 gene, encoding the $\beta 2$-subunit of AMP-activated protein kinase, may also have contributed to the cardiac phenotype observed in our patient. PRKAB2 is highly expressed in cardiac muscle. Mutations in the gene encoding the AMPK $\gamma 2$-subunit (PRKAG2) have been shown to cause hypertrophic cardiomyopathy [Blair et al., 2001]. However, no pathogenic PRKAB2 mutations have been described in humans so far.

The extreme phenotypic variation observed in our family suggests that additional genetic or environmental factors modify the clinical phenotype, resembling the 
proposed 2-hit model for severe DD/ID in 16p12.1 microdeletion carriers [Girirajan et al., 2010]. Furthermore, our report underlines the importance of family studies in hitherto poorly defined microdeletion and microduplication syndromes.

\section{Acknowledgements}

We are grateful to the proband and her family members for their participation in this study. We thank Tom de Vries Lentsch for his assistance with the illustrations and Hellen de Bruin-van der Wende for administrative support.

\section{References}

Albers CA, Paul DS, Schulze H, Freson K, Stephens JC, et al: Compound inheritance of a low-frequency regulatory SNP and a rare null mutation in exon-junction complex subunit RBM8A causes TAR syndrome. Nat Genet 44 435-439 (2012).

Blair E, Redwood C, Ashrafian H, Oliveira M, Broxholme J, et al: Mutations in the gam$\mathrm{ma}(2)$ subunit of AMP-activated protein kinase cause familial hypertrophic cardiomyopathy: evidence for the central role of energy compromise in disease pathogenesis. Hum Mol Genet 10:1215-1220 (2001).

Brunetti-Pierri N, Berg JS, Scaglia F, Belmont J, Bacino CA, et al: Recurrent reciprocal 1q21.1 deletions and duplications associated with microcephaly or macrocephaly and developmental and behavioral abnormalities. Nat Genet 40:1466-1471 (2008).

Cheroki C, Krepischi-Santos AC, Szuhai K, Brenner V, Kim CA, et al: Genomic imbalances associated with Mullerian aplasia. J Med Genet 45:228-232 (2008).

de Leeuw N, Hehir-Kwa JY, Simons A, Geurts van Kessel A, Smeets DF, et al: SNP array analysis in constitutional and cancer genome diagnostics - copy number variants, genotyping and quality control. Cytogenet Genome Res 135 212-221 (2011)

Digilio MC, Bernardini L, Consoli F, Lepri FR, Giuffrida MG, et al: Congenital heart defects in recurrent reciprocal 1q21.1 deletion and duplication syndromes: rare association with pulmonary valve stenosis. Eur J Med Genet 56:144-149 (2013).
Dolcetti A, Silversides CK, Marshall CR, Lionel AC, Stavropoulos DJ, et al: 1q21.1 microduplication expression in adults. Genet Med 15: 282-289 (2013).

Girirajan S, Rosenfeld JA, Cooper GM, Antonacci F, Siswara P, et al: A recurrent 16p12.1 microdeletion supports a two-hit model for severe developmental delay. Nat Genet 42:203209 (2010).

Gollob MH, Jones DL, Krahn AD, Danis L, Gong $\mathrm{XQ}$, et al: Somatic mutations in the connexin 40 gene (GJA5) in atrial fibrillation. N Engl J Med 354:2677-2688 (2006).

Greenway SC, Pereira AC, Lin JC, DePalma SR, Israel SJ, et al: De novo copy number variants identify new genes and loci in isolated sporadic tetralogy of Fallot. Nat Genet 41:931935 (2009).

Gu H, Smith FC, Taffet SM, Delmar M: High incidence of cardiac malformations in connexin40-deficient mice. Circ Res 93:201-206 (2003).

Guida V, Ferese R, Rocchetti M, Bonetti M, Sarkozy A, et al: A variant in the carboxyl-terminus of connexin 40 alters GAP junctions and increases risk for tetralogy of Fallot. Eur J Hum Genet 21:69-75 (2012).

Kirchhoff S, Kim JS, Hagendorff A, Thonnissen E, Kruger O, et al: Abnormal cardiac conduction and morphogenesis in connexin 40 and connexin43 double-deficient mice. Circ Res 87: 399-405 (2000).
Klopocki E, Schulze H, Strauss G, Ott CE, Hall J, et al: Complex inheritance pattern resembling autosomal recessive inheritance involving a microdeletion in thrombocytopenia-absent radius syndrome. Am J Hum Genet 80:232240 (2007).

Mefford HC, Sharp AJ, Baker C, Itsara A, Jiang $Z$, et al: Recurrent rearrangements of chromosome 1q21.1 and variable pediatric phenotypes. N Engl J Med 359:1685-1699 (2008).

Rosenfeld JA, Traylor RN, Schaefer GB, McPherson EW, Ballif BC, et al: Proximal microdeletions and microduplications of 1q21.1 contribute to variable abnormal phenotypes. Eur J Hum Genet 20:754-761 (2012).

Soemedi R, Topf A, Wilson IJ, Darlay R, Rahman T, et al: Phenotype-specific effect of chromosome 1q21.1 rearrangements and GJA5 duplications in 2436 congenital heart disease patients and 6760 controls. Hum Mol Genet 21: 1513-1520 (2012).

Wirka RC, Gore S, Van Wagoner DR, Arking DE, Lubitz SA, et al: A common connexin-40 gene promoter variant affects connexin-40 expression in human atria and is associated with atrial fibrillation. Circ Arrhythm Electrophysiol 4:87-93 (2011).

Yang YQ, Liu X, Zhang XL, Wang XH, Tan HW, et al: Novel connexin40 missense mutations in patients with familial atrial fibrillation. Europace 12:1421-1427 (2010). 